\title{
Current and Future Treatment Strategies for Patients with Advanced Hepatocellular Carcinoma: Role of mTOR Inhibition
}

\author{
Richard S. Finn \\ Division of Hematology/Oncology, David Geffen School of Medicine, \\ University of California, Los Angeles, CA, USA
}

\section{Key Words}

Everolimus · Hepatocellular carcinoma $\cdot$ Immunosuppression $\cdot$ mTOR inhibition · Sirolimus

\begin{abstract}
Hepatocellular carcinoma (HCC) is a common cancer that has the third highest cancer-related mortality rate worldwide. Although potentially curable by transplantation if detected early, the majority of cases are diagnosed at an advanced stage of disease for which limited treatment options are available. The only proven systemic therapy for advanced HCC is sorafenib, a multi-kinase inhibitor that has demonstrated modest efficacy and reasonable tolerability in patients with advanced HCC. Five years after the approval of sorafenib, no other agent has been proven to be beneficial in the first- or second-line setting in advanced HCC. While molecular studies have highlighted various potential targets in HCC, the mammalian target of rapamycin (mTOR) has emerged as an exciting target for cancer therapy including HCC. Laboratory data have linked the phosphatidylinositol 3-kinase/AKT/mTOR axis to various oncogenic processes, including survival and angiogenesis. Historically, mTOR inhibitors have been used for their immunosuppressive properties, but more recently they have been approved as anticancer agents. Retrospective HCC studies suggest that the inclusion of mTOR inhibition as part of an immunosuppressant regimen after transplantation may reduce HCC recurrence compared with other immunosuppressive agents such as calcineurin inhibitors. More recently, single-arm, phase I/II studies have shown that mTOR inhibitors also have activity as monotherapy in cases of recurrent HCC or de novo advanced HCC. This article will review the rationale for targeting the mTOR pathway in HCC, and the currently available clinical data supporting its development for HCC.

Copyright (C) 2012 S. Karger AG, Basel
\end{abstract}

Richard S. Finn, MD

Division of Hematology/Oncology, David Geffen School of Medicine, University of California, Los Angeles

10833 Le Conte Avenue, 11-0934 Factor Building, Los Angeles, CA 90095 (USA)

Tel. +1 310586 2091, E-mail rfinn@mednet.ucla.edu 


\section{Introduction}

Hepatocellular carcinoma (HCC) accounts for $70-85 \%$ of primary liver cancers and is the sixth most common form of cancer worldwide [1-3]. Because of its high ratio of mortality to incidence (0.93), liver cancer is the third most frequent cause of cancer-related death $[1,2]$. In its earliest stages, HCC is frequently asymptomatic and goes undetected, resulting in almost $85 \%$ of patients being diagnosed at intermediate or advanced stages when curative therapies such as surgical resection or liver transplantation are no longer an option [4, 5]. Even when patients present with early-stage disease and surgical resection is an option, $70 \%$ of such patients experience recurrence of the original tumor or develop new tumors within 5 years [6]. Similarly, patients with early-stage disease who are not amenable to surgery and receive locoregional treatments such as radiofrequency ablation or transarterial chemoembolization almost always experience recurrence and require additional therapy [5]. The fact that HCC almost always occurs in the presence of underlying liver disease adds an additional layer of complexity to its management. For patients who are not eligible for potentially curative treatment, locoregional and systemic therapies may improve survival for selected patients. For most patients, if they do not die from underlying cirrhosis, their tumor will progress to the point where additional therapy is needed. For patients who progress from intermediate- to advanced-stage disease, the multi-targeted tyrosine kinase inhibitor sorafenib has been proven to extend survival $[7,8]$. However, for patients who are intolerant of or progress on sorafenib, there exists a large unmet need as no other agent has been shown to be effective in these settings.

\section{Systemic Therapy for Advanced HCC}

Sorafenib is the only systemic therapy approved for the treatment of advanced HCC. The approval of sorafenib in this setting was based on the significant improvement in overall survival and manageable toxicity profile observed in two randomized, placebo-controlled, phase III studies, the sorafenib hepatocellular carcinoma assessment randomized protocol (SHARP) study conducted in North and South America, Australia, and Europe [7] and a similar trial conducted in Asia [8]. In SHARP, median overall survival was improved by 2.8 months with sorafenib compared with placebo (10.7 months vs. 7.9 months; hazard ratio [HR], 0.69; 95\% confidence interval [CI], 0.55-0.87; $\mathrm{p}<0.001$ ] [7]. In the Asia-Pacific trial, median overall survival was improved by 2.3 months with sorafenib compared with placebo (6.5 months vs. 4.2 months; HR, 0.68; 95\% CI, 0.50-0.93; p = 0.014) [8]. In both trials, the response rate was low (partial response rates of 2 and $3 \%$, respectively), but the median time to progression was improved from 2.8 months to 5.5 months $(\mathrm{p}<0.001)$ in SHARP and from 1.4 months to 2.8 months in the Asia-Pacific study [7, 8]. These studies established sorafenib as the standard of care for this population, but now, 5 years later, it is readily apparent that new therapeutic options are needed. In that regard, molecular targeted therapeutics lead the landscape in clinical development, largely driven by the lack of efficacy of cytotoxic agents in the treatment of HCC [5]. Furthermore, recent phase III data from a second-line study of the VEGFr-/fibroblast growth factor receptor (FGFr)-TKI brivanib [9] suggest that the natural history of advanced HCC after progression with sorafenib has changed.

The range of novel agents under development for advanced HCC is impressive. There are currently several phase II studies, as well as randomized phase III studies, evaluating single-agent or combination activity in HCC. The challenge for clinical development in HCC is two-fold: (1) designing trials that select a population of patients whose overall medical condition due to underlying liver disease is not too severe, and (2) identifying which new 
targets and agents are most promising for further development. In addition, the identification of a predictive marker associated with response is always preferred in clinical development, as opposed to an approach that does not preselect patients that are more likely to benefit from a given therapy. While predictive biomarkers exist for certain cancers (e.g., HER2 amplification in breast cancer and EGFR mutations in lung cancer), there are no validated targets there are no validated targets with associated predictive markers in HCC.

Several molecular targets are being pursued in HCC, including growth factors associated with angiogenesis (VEGF and FGF), the epidermal growth factor receptor (EGFR), the hepatocyte growth factor and its receptor (HGFR/c-Met), the insulin-like growth factor receptor (IGFR), the ras/raf/mitogen-activated protein kinase (MAPK) pathway, survivin (negative regulator of apoptosis), Wnt signaling, Src signaling, and the phosphatidylinositol 3-kinase (PI3 K)/AKT/mammalian target of the rapamycin (mTOR) pathway [5, 10, 11].Of these targets, both mTOR and the VEGF receptor are currently undergoing evaluation as second-line therapy for HCC in phase III studies. This review will focus on the laboratory and clinical data that support the target mTOR in regard to HCC.

\section{The mTOR Signaling Pathway and HCC}

mTOR is a serine/threonine kinase that regulates cell growth, proliferation, survival, metabolism, and angiogenesis by integrating signals received from the PI3 K/Akt signaling pathway (fig. 1) [12]. mTOR occurs in two multiprotein complexes (mTORC1 and mTORC2), of which mTORC1 is the target of the macrolide antibiotic rapamycin (sirolimus) and its analogs everolimus, temsirolimus, and ridaforolimus. Aside from mTOR, the mTORC1 complex includes the regulatory-associated protein of mTOR (raptor), mammalian LST8 (mLST8, also called G $\beta \mathrm{L}$ ), and the proline-rich Akt substrate 40kDa (PRAS40). mTORC1 activity is responsive to available nutrients, energy, and growth factors in the cell, and regulates the protein expression necessary for cell metabolism, growth, proliferation, and angiogenesis [12]. Aside from PI3 K and Akt, other key signaling molecules upstream of mTORC1 include the Rag guanosine triphosphatases (GTPases), adenosine monophosphate kinase (AMPK), tuberous sclerosis complex (TSC) proteins TSC1 (hamartin) and TSC2 (tuberin), and the phosphatase and tensin homolog (PTEN). Direct targets of mTORC1 include ribosomal protein S6 kinase 1 (S6K1) and the eukaryotic protein synthesis initiation factor 4E-binding protein 1 (4E-BP1), both of which regulate protein translation [12].

Several lines of evidence implicate mTOR signaling in hepatocarcinogenesis. In a HCC mouse model, chronic activation of mTORC1 was found to be associated with hepatocyte damage and development of HCC through a pathological program of liver damage and inflammation [13]. In approximately $40-50 \%$ of human HCC tumors, dysregulated expression of both up- and downstream effectors of mTOR, including EGF, IGFBP3, IGF2, raptor, PTEN, RPS6, and p70S6 kinase, has been observed [14-16]. Furthermore, activated mTOR signaling was found to be associated with poor prognosis in HCC patients [16]. In an analysis of HCC patients treated by surgical resection, those whose tumors expressed phosphorylated RPS6 (pRPS6) had a significantly shorter median time to recurrence than those with pRPS6-negative tumors (25 months vs. 50 months; $p=0.004$ ), and expression of pRPS6 was an independent predictor of recurrence (HR, 1.8; 95\% CI, 1.1-2.8; $\mathrm{p}=0.01$ ) [16]. 
Fig. 1. The mTOR signaling pathway. Reproduced with permission from Al-Batran et al. [50].

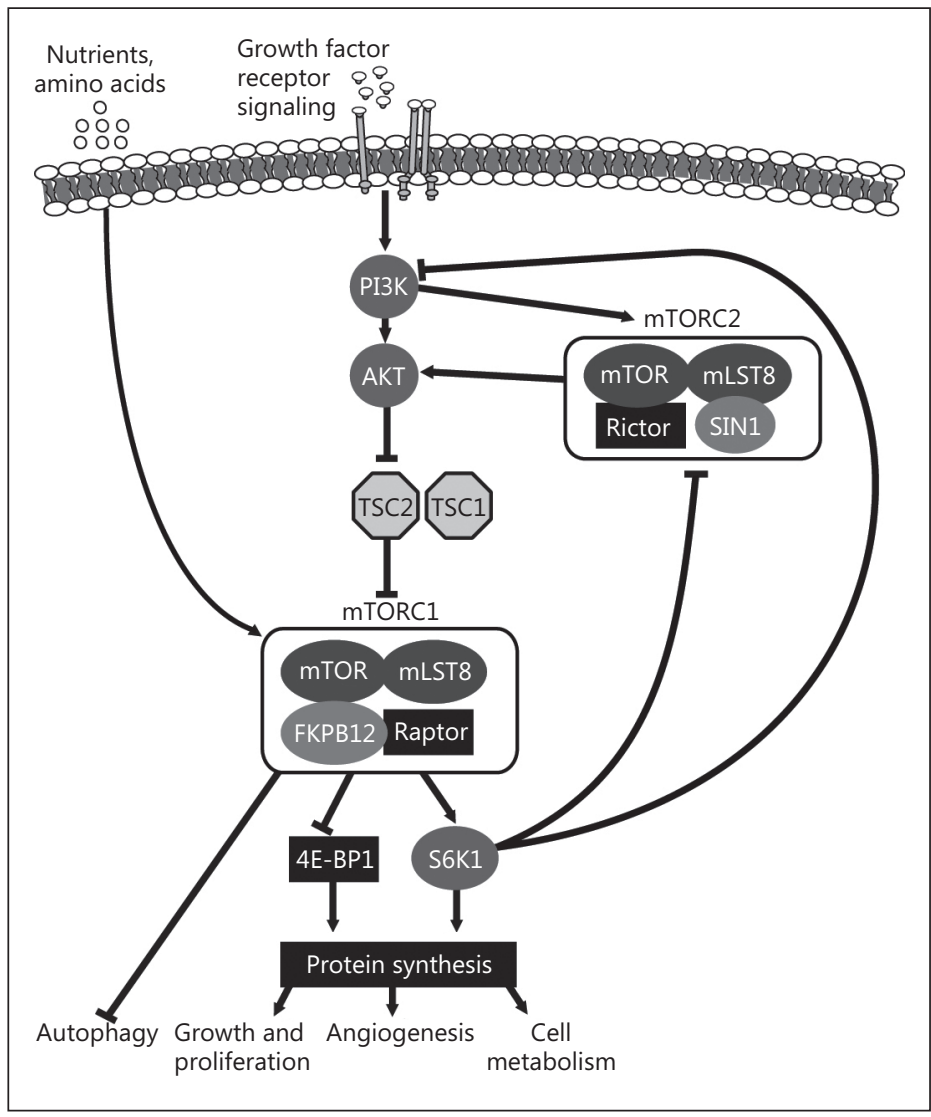

\section{mTOR Inhibitors in HCC}

\section{Supportive preclinical evidence}

The biologic rationale for the role of mTOR in the pathogenesis of HCC is supported by several preclinical studies. In human HCC cell lines, everolimus, sirolimus, and temsirolimus have been shown to inhibit cell growth and proliferation [14, 16-19]. In various HCC mouse and rat models, everolimus and sirolimus significantly reduced tumor volume and angiogenesis, delayed tumor growth, and increased overall survival compared with control [16-20]. For example, in a mouse xenograft model, treatment with everolimus ( $5 \mathrm{mg} / \mathrm{kg}$ ) significantly reduced tumor growth at 15 days compared with placebo $\left(1039 \mathrm{~mm}^{3} \mathrm{vs} .2396 \mathrm{~mm}^{3} ; \mathrm{p}<\right.$ 0.05 ) and significantly increased median overall survival (19 days vs. 16 days, $p<0.05$ ) [16]. Additional preclinical evidence supports the exploration of mTOR inhibitors in combination with agents targeting other signaling pathways, such as VEGF $[19,21]$. In one study, everolimus and sorafenib synergistically inhibited proliferation in HCC cell lines and tumor growth in HCC xenografts [19].

\section{mTOR inhibitors and recurrence of HCC after liver transplantation}

HCC is the only solid tumor with a potentially high rate of cure by organ transplantation in selected patients [6]. Currently, HCC recurrence following transplantation represents a therapeutic challenge. Case reports and retrospective analyses suggest that in addition to its proven activity in advanced HCC in the non-transplantation setting, sorafenib may have activity in patients with post-transplantation recurrence [22] and may be tolerable in the adjuvant setting [23]. Other retrospective studies have suggested that the risk of HCC recur- 
rence is increased when calcineurin inhibitors (CNIs) such as cyclosporine and tacrolimus are included as part of the post-transplantation immunosuppression regimen [24-26]. The exact mechanism for this increased risk is unknown, although preclinical data suggest that CNIs may actually promote tumor growth $[27,28]$. In contrast, other data suggest that immunosuppression regimens that include rapamycin (sirolimus) or its analogs reduce the risk of HCC recurrence as well as the development of de novo malignancies after orthotopic liver transplantation, while successfully avoiding allograft rejection and improving overall survival [24-26, 29-34]. A recent meta-analysis showed that compared with sirolimus-free immunosuppression post-transplantation, sirolimus-based immunosuppression in patients with HCC significantly improved overall survival at 1 year (odds ratio [OR], 4.53; 95\% CI, 2.31-8.89), 3 years (OR, 1.97; 95\% CI, 1.29-3.00), and 5 years (OR, 2.47; 95\% CI, 1.72-3.55) and decreased tumor recurrence (OR, 0.42; 95\% CI, 0.21-0.83) [29]. Moreover, preclinical data suggest that immunosuppressive and antitumor activity can be achieved simultaneously at the target trough levels of the mTOR inhibitor used for immunosuppression [35].The exact mechanism of decreased HCC recurrence observed with mTOR inhibitor-based immunosuppression in liver transplantation recipients is unknown. It could be the result of modification of the degree of immunosuppression, representative of a direct anticancer effect, or a combination of both.

In addition, two separate case reports describe patients who experienced either partial or complete radiological response after receiving sirolimus and sorafenib [36, 37]. A recent retrospective analysis of 26 transplantation recipients who had received sorafenib and either everolimus or sirolimus following recurrence showed an overall response rate of $3.8 \%$, a stable disease rate of $50.0 \%$, a median time to progression of 6.8 months ( $95 \%$ CI, 2.3-11.1 months), and a median overall survival of 19.3 months (95\% CI, 13.4-25.1 months) [38]. The authors of this study concluded that the combination had a "significant but manageable toxicity profile." The authors of a second, smaller retrospective analysis $(n=13)$ also found the combination to have promising efficacy but considered the toxicity profile to be more significant [39]. Appropriately controlled prospective studies are needed to fully evaluate the clinical benefit of combination therapy with sorafenib and mTOR inhibitor-based immunosuppression in HCC patients who experience recurrence after liver trancesplation.

\section{mTOR inhibition in unresectable advanced HCC}

Early pilot trials conducted in Europe on mTOR inhibition for patients with advanced HCC showed limited efficacy for sirolimus, with only one partial response among the 39 patients enrolled and median overall survival of 5.3-6.5 months [40, 41]. Encouragingly, sirolimus was well tolerated and the safety profile was manageable. A phase II study of weekly sirolimus (20 mg/week for 1 month then $30 \mathrm{mg} /$ week) that enrolled 25 treatment-naive patients with advanced HCC demonstrated similar outcomes [42]. Among the 23 evaluable patients, the best overall response was complete and partial response in one patient each and stable disease in 8 patients. Median overall survival was 6.1 months (range, 1.9-40.1 months).

The rapamycin analog everolimus has also been studied in patients with advanced HCC. Currently, everolimus has proven anticancer activity in advanced renal cell carcinoma following VEGFr-TKIs [43], in advanced, progressive neuroendocrine tumors [44], and, in combination with exemestane, in advanced hormone receptor-positive, human epidermal growth factor receptor 2-negative breast cancer that progressed on prior hormonal therapy [45]. Data regarding the safety and efficacy of everolimus in HCC come from two prospective studies [46, 47]. In a phase I/II trial conducted in 28 patients with advanced HCC, 20 (71\%) of whom had received previous systemic therapy, no dose-limiting toxicities were experienced and everolimus was generally well tolerated at a dose of $10 \mathrm{mg} /$ day [46]. Among the 25 patients treated with everolimus at $10 \mathrm{mg} /$ day, the best overall response was partial response in one patient. 
An additional 10 patients experienced stable disease of $\geq 12$ weeks duration, and median overall survival was 8.4 months (95\% CI, 3.9-21.1 months) [46]. A phase I dose-finding study on everolimus for advanced HCC evaluated both daily $(n=21)$ and weekly $(n=18)$ dosing schedules [47]. Based on the observed dose-limiting toxicities of hyperbilirubinemia, alanine aminotransferase elevation, thrombocytopenia, infection, diarrhea, rectal bleeding, and cardiac ischemia, the maximum tolerated doses were determined to be $70 \mathrm{mg} / \mathrm{week}$ and $7.5 \mathrm{mg} /$ day. Out of 39 evaluable patients, there was one partial response and 21 patients with stable disease (daily, 71\%; weekly, 44\%). Median overall survival was 7.7 months (95\% CI, 2.1-13.3 months) and 5.7 months (95\% CI, 0-12.3 months) for daily and weekly dosing, respectively. Reactivation of hepatitis B virus (HBV) was observed in 5 of 27 patients (18\%) who were seropositive for hepatitis B antigen, which suggests that prophylactic antiviral therapy should be used in patients with HBV [47].

Based on the scientific rationale supporting the activity of mTOR in HCC, the results of two clinical trials, and the unmet needs of patients who cannot tolerate or progress on sorafenib, the international phase III everolimus for liver cancer evaluation (EVOLVE-1) study was initiated (table 1$)$. In this study, the efficacy and safety of everolimus $(7.5 \mathrm{mg} /$ day plus best supportive care) is now being compared with that of placebo plus best supportive care in either those patients with advanced HCC and whose disease has progressed following sorafenib treatment or those who cannot tolerate sorafenib. This study has now completed enrolment, and results are pending. In addition to everolimus, there are ongoing trials of the mTOR inhibitor temsirolimus monotherapy in patients with advanced HCC (table 1).

Since sorafenib is the current first-line standard of care for advanced HCC, agents introduced in the first-line setting would either have to prove single-agent superiority over sorafenib or provide additional activity when combined with it. The first phase III study of combination therapy in advanced HCC was SEARCH, a randomized trial protocol for the treatment of patients with hepatocellular carcinoma by sorafenib plus the EGFr-TKI erlotinib. Recently published results from this study show that the combination of sorafenib and erlotinib did not significantly improve survival over sorafenib alone [48]. However, the scientific rationale for this combination was not very well established. As discussed above, the mTOR axis has laboratory data to support its role in the pathogenesis of HCC and singleagent studies suggesting clinical activity. Preclinical studies conducted in HCC models have evaluated the combination of rapamycin and its analogs with sorafenib $[19,21]$. There are numerous trials underway to investigate the use of mTOR inhibitors in combination with other agents, including the VEGF inhibitor bevacizumab, the VEGFr-TKI sorafenib, and more traditional chemotherapeutic agents such as doxorubicin, as well as in combination with locoregional therapy (table 1). Based on currently available data, it is unclear whether combination therapy with mTOR inhibitors and VEGF-targeted agents will have an acceptable tolerability profile $[36,38,39,49]$. In one dose-finding study of combination therapy with everolimus and sorafenib, the maximum tolerated dose of everolimus given with standarddose sorafenib (400 mg twice daily) was $2.5 \mathrm{mg}$ once daily, which was not considered to be a biologically effective dose [49]. Ongoing studies may provide a clearer picture of the relative balance between efficacy and safety for mTOR-based combination therapy in advanced HCC (table 1).

\section{Summary and Future Directions}

The approval of sorafenib has changed the clinical landscape of advanced HCC. However, despite this advance, no new agents have been approved since. While several novel targets 


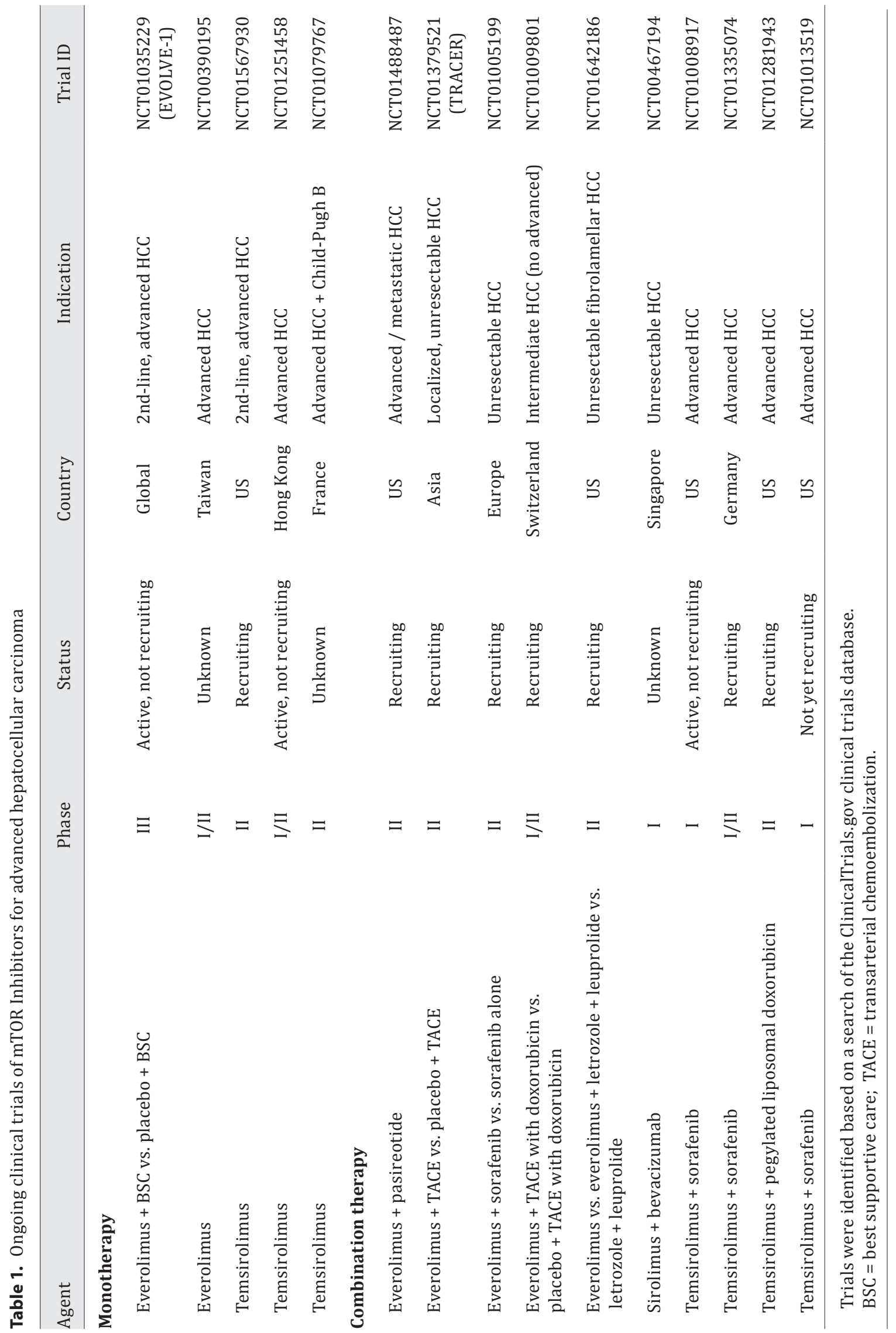


of interest are being pursued in clinical studies, everolimus is currently the only orally bioavailable mTOR inhibitor approved for cancer treatment, and its development is the most advanced in regard to HCC. Several lines of evidence support the development of mTOR inhibitors for HCC, including the pivotal role of mTOR in cancer biology, data suggesting its activation is important in HCC, and uncontrolled clinical data suggesting that mTOR inhibitors have single-agent clinical activity. Aside from its potential role in the treatment of advanced HCC, retrospective data suggest that mTOR inhibitor-based immunosuppression may play a role in managing the risk of HCC recurrence following orthotopic liver transplantation. However, to validate this hypothesis, appropriately powered prospective studies are needed. In addition, the potential role for mTOR inhibition in combination with other targeted therapies is under evaluation. Ultimately, as with all targeted agents, a predictive marker for patient selection would aid in the identification of patients most likely to respond to everolimus and other mTOR inhibitors. Until such a biomarker is identified, data from current clinical investigations will help define the role of targeting mTOR in HCC.

\section{Acknowledgments}

RSF has received support from the Auerbach Family Gift for Emerging Therapies in Hepatocellular Carcinoma and the Pfleger Foundation. Editorial assistance in support of this manuscript was provided by Alison Comer, PhD (ApotheCom, Yardley, PA, USA), and was funded by Novartis Pharmaceuticals.

\section{Conflicts of Interest}

The author has served as a consultant to Onyx, Bayer, Bristol-Myers Squibb, and Novartis.

\section{References}

1 Ferlay J, Shin HR, Bray F, et al: Estimates of worldwide burden of cancer in 2008: GLOBOCAN 2008. Int J Cancer 2010;127:2893-2917.

2 Jemal A, Bray F, Center MM, et al: Global cancer statistics, 2011. CA Cancer J Clin 2011;61:69-90.

3 El-Serag HB: Epidemiology of hepatocellular carcinoma in USA. Hepatol Res 2007;37:S88-S94.

4 Villanueva A, Llovet JM: Targeted therapies for hepatocellular carcinoma. Gastroenterology 2011;140: 1410-1426.

5 Finn RS: Development of molecularly targeted therapies in hepatocellular carcinoma: where do we go now? Clin Cancer Res 2010;16:390-397.

6 Forner A, Llovet JM, Bruix J: Hepatocellular carcinoma. Lancet 2012;379:1245-1255.

7 Llovet JM, Ricci S, Mazzaferro V, et al: Sorafenib in advanced hepatocellular carcinoma. N Engl J Med 2008;359:378-390.

8 Cheng AL, Kang YK, Chen Z, et al: Efficacy and safety of sorafenib in patients in the Asia-Pacific region with advanced hepatocellular carcinoma: a phase III randomised, double-blind, placebo-controlled trial. Lancet Oncol 2009;10:25-34.

9 Llovet JM, Decaens T, Raoul JL, et al: Brivanib versus placebo in patients with advanced hepatocellular carcinoma (HCC) who failed or were intolerant to sorafenib: results from the phase 3 BRISK-PS study. Presented at the 2012 International Liver Congress; April 18-22, 2012; Barcelona, Spain.

10 Aravalli RN, Steer CJ, Cressman EN: Molecular mechanisms of hepatocellular carcinoma. Hepatology 2008;48:2047-2063.

11 Bhoori S, Toffanin S, Sposito C, et al: Personalized molecular targeted therapy in advanced, recurrent hepatocellular carcinoma after liver transplantation: a proof of principle. J Hepatol 2010;52:771-775.

12 Fasolo A, Sessa C: Targeting mTOR pathways in human malignancies. Curr Pharm Des 2012;18:27662777.

13 Menon S, Yecies JL, Zhang HH, et al: Chronic activation of mTOR complex 1 is sufficient to cause hepatocellular carcinoma in mice. Sci Signal 2012;5:ra24. 
14 Sahin F, Kannangai R, Adegbola O, et al: mTOR and P70 S6 kinase expression in primary liver neoplasms. Clin Cancer Res 2004;10:8421-8425.

15 Sieghart W, Fuereder T, Schmid K, et al: Mammalian target of rapamycin pathway activity in hepatocellular carcinomas of patients undergoing liver transplantation. Transplantation 2007;83:425-432.

16 Villanueva A, Chiang DY, Newell P, et al: Pivotal role of mTOR signaling in hepatocellular carcinoma. Gastroenterology 2008;135:1972-1983.

17 Thomas HE, Mercer CA, Carnevalli LS, et al: mTOR Inhibitors synergize on regression, reversal of gene expression, and autophagy in hepatocellular carcinoma. Sci Transl Med 2012;4:139ra84.

18 Semela D, Piguet AC, Kolev M, et al: Vascular remodeling and antitumoral effects of mTOR inhibition in a rat model of hepatocellular carcinoma. J Hepatol 2007;46:840-848.

19 Piguet AC, Saar B, Hlushchuk R, et al: Everolimus augments the effects of sorafenib in a syngeneic orthotopic model of hepatocellular carcinoma. Mol Cancer Ther 2011;10:1007-1017.

20 Huynh H, Chow KH, Soo KC, et al: RAD001 (everolimus) inhibits tumour growth in xenograft models of human hepatocellular carcinoma. J Cell Mol Med 2009;13:1371-1380.

21 Newell P, Toffanin S, Villanueva A, et al: Ras pathway activation in hepatocellular carcinoma and anti-tumoral effect of combined sorafenib and rapamycin in vivo. J Hepatol 2009;51:725-733.

22 Yeganeh M, Finn RS, Saab S: Apparent remission of a solitary metastatic pulmonary lesion in a liver transplant recipient treated with sorafenib. Am J Transplant 2009;9:2851-2854.

23 Saab S, McTigue M, Finn RS, et al: Sorafenib as adjuvant therapy for high-risk hepatocellular carcinoma in liver transplant recipients: feasibility and efficacy. Exp Clin Transplant 2010;8:307-313.

24 Chinnakotla S, Davis GL, Vasani S, et al: Impact of sirolimus on the recurrence of hepatocellular carcinoma after liver transplantation. Liver Transpl 2009;15:1834-1842.

25 Toso C, Merani S, Bigam DL, et al: Sirolimus-based immunosuppression is associated with increased survival after liver transplantation for hepatocellular carcinoma. Hepatology 2010;51:1237-1243.

26 Zimmerman MA, Trotter JF, Wachs M, et al: Sirolimus-based immunosuppression following liver transplantation for hepatocellular carcinoma. Liver Transpl 2008;14:633-638.

27 Freise CE, Ferrell L, Liu T, et al: Effect of systemic cyclosporine on tumor recurrence after liver transplantation in a model of hepatocellular carcinoma. Transplantation 1999;67:510-513.

28 Schnitzbauer AA, Zuelke C, Graeb C, et al: A prospective randomised, open-labeled, trial comparing sirolimus-containing versus mTOR-inhibitor-free immunosuppression in patients undergoing liver transplantation for hepatocellular carcinoma. BMC Cancer 2010;10:190.

29 Liang W, Wang D, Ling X, et al: Sirolimus-based immunosuppression in liver transplantation for hepatocellular carcinoma: a meta-analysis. Liver Transpl 2012;18:62-69.

30 Mártinez JM, Pulido LB, Bellido CB, et al: Rescue immunosuppression with mammalian target of rapamycin inhibitor drugs in liver transplantation. Transplant Proc 2010;42:641-643.

31 Gomez-Camarero J, Salcedo M, Rincon D, et al: Use of everolimus as a rescue immunosuppressive therapy in liver transplant patients with neoplasms. Transplantation 2007;84:786-791.

32 Vivarelli M, Dazzi A, Cucchetti A, et al: Sirolimus in liver transplant recipients: a large single-center experience. Transplant Proc 2010;42:2579-2584.

33 Perrakis A, Schwarz K, Yedibela S, et al: Impact of the conversion of the immunosuppressive regimen from prograf to advagraf or to sirolimus in long-term stable liver transplant recipients: indications, safety, and outcome. Transplant Proc 2011;43:3702-3707.

34 Bilbao I, Sapisochin G, Dopazo C, et al: Indications and management of everolimus after liver transplantation. Transplant Proc 2009;41:2172-2176.

35 Guba M, von Breitenbuch P, Steinbauer M, et al: Rapamycin inhibits primary and metastatic tumor growth by antiangiogenesis: involvement of vascular endothelial growth factor. Nat Med 2002;8:128-135.

36 Wang Y, Speeg KV, Washburn WK, et al: Sirolimus plus sorafenib in treating HCC recurrence after liver transplantation: a case report. World J Gastroenterol 2010;16:5518-5522.

37 Kim R, Aucejo F: Radiologic complete response with sirolimus and sorafenib in a hepatocellular carcinoma patient who relapsed after or thotopic liver transplantation. J Gastrointest Cancer 2011;42:50-53.

38 Gomez-Martin C, Bustamante J, Castroagudin JF, et al: Efficacy and safety of sorafenib in combination with mammalian target of rapamycin inhibitors for recurrent hepatocellular carcinoma after liver transplantation. Liver Transpl 2012;18:45-52.

39 Staufer K, Fischer L, Seegers B, et al: High toxicity of sorafenib for recurrent hepatocellular carcinoma after liver transplantation. Transpl Int 2012;25:1158-1164.

40 Rizell M, Andersson M, Cahlin C, et al: Effects of the mTOR inhibitor sirolimus in patients with hepatocellular and cholangiocellular cancer. Int J Clin Oncol 2008;13:66-70.

41 Schöniger-Hekele M, Muller C: Pilot study: rapamycin in advanced hepatocellular carcinoma. Aliment Pharmacol Ther 2010;32:763-768.

42 Decaens T, Luciani A, Itti E, et al: Phase II study of sirolimus in treatment-naive patients with advanced hepatocellular carcinoma. Dig Liver Dis 2012;44:610-616.

43 Motzer RJ, Escudier B, Oudard S, et al: Phase 3 trial of everolimus for metastatic renal cell carcinoma: final results and analysis of prognostic factors. Cancer 2010;116:4256-4265.

44 Yao JC, Shah MH, Ito T, et al: Everolimus for advanced pancreatic neuroendocrine tumors. N Engl J Med 2011;364:514-523.

45 Baselga J, Campone M, Piccart M, et al: Everolimus in postmenopausal hormone receptor-positive advanced breast cancer. N Engl J Med 2012;366:520-529. 
46 Zhu AX, Abrams TA, Miksad R, et al: Phase 1/2 study of everolimus in advanced hepatocellular carcinoma. Cancer 2011;117:5094-5102.

47 Shiah HS, Chen CY, Hsiao CF, et al: Randomised clinical trial: comparison of two everolimus dosing schedules in patients with advanced hepatocellular carcinoma. Aliment Pharmacol Ther 2012 Nov 8. doi: 10.1111/apt.12132. [Epub ahead of print]

48 Zhu AX, Rosmorduc 0, Evans J, et al: A phase III randomized, double-blind, placebo-controlled trial of sorafenib plus erlotinib in patients with hepatocellular carcinoma (HCC). Presented at the 2012 Congress of the European Society of Medical Oncology; September 28-October 2, 2012; Vienna, Austria.

49 Finn RS, Poon RTP, Yau T, et al: Phase I study of everolimus in combination with sorafenib in patients with advanced hepatocellular carcinoma. J Clin Oncol 29: 2011 (suppl; abstr 4074).

50 Al-Batran SE, Ducreaux M, Ohtsu A: mTOR as a therapeutic target in patients with gastric cancer. Int J Cancer 2012;130:491-496. 